\section{Nutrition Support for Athletes}

$\mathrm{T}$

The Ryley-Jeffs Memorial lecture given by Kelly Anne Erdman at Dietitians of Canada's 2015 conference titled A

Lifetime Pursuit of a Sport Nutrition Practice and profiled in this issue, gives us a "behind the scenes" look at the world of sports nutrition. After the establishment of the National Sport Centre Calgary, Kelly Anne practiced as the first consulting dietitian for several Canadian national teams, including Hockey Canada. Career highlights have included the opportunity to work at 3 Games for the Canadian Olympic Committee as the Performance Dietitian for Team Canada athletes (2011 Pan American Games, 2012 London Summer Olympics, and the 2014 Sochi Winter Olympics). This involved ensuring that the Canadian athletes had ready access to safe and effective foods to perform at their best. Kelly Anne describes ongoing challenges in sport dietetics, including dealing with advice given to athletes by pseudo-nutritionists, and also the tremendous opportunities available for aspiring Canadian sport dietitians.

As an honoured Ryley-Jeffs award recipient, Kelly Anne's visionary leadership and pioneering spirit in the field of sport nutrition, certainly exemplifies the vision of Violet Ryley and Kathleen Jeffs. Congratulations Kelly Anne!

Check the current issue of the Journal for the complete CFDR Dietetic Research Event Abstracts from this year's conference.

(Can J Diet Pract Res. 2015; 76:3)

(DOI: $10.3148 /$ cjdpr-2015-023)

Published at dcjournal.ca on 17 August 2015.

\section{Du soutien nutritionnel pour les athlètes}

$\mathrm{L}$ a conférence commémorative RyleyJeffs de cette année, prononcée par Kelly Anne Erdman lors du congrès 2015 des Diététistes du Canada et intitulée A Lifetime Pursuit of a Sport Nutrition Practice [La pratique de la nutrition du sport : la passion d'une vie], lève le voile sur le monde de la nutrition du sport. Vous pourrez la lire dans le présent numéro de la Revue. Après l'établissement du National Sport Centre Calgary, Kelly Anne y a établi sa pratique à titre de première diététisteconsultante pour plusieurs équipes nationales canadiennes, entre autres Hockey Canada. Au cours de sa carrière, elle a eu la chance d'occuper au sein du Comité olympique canadien le poste de diététiste du sport pour les athlètes d'Équipe Canada dans le cadre de trois jeux (Jeux panaméricains de 2011, Jeux olympiques d'été de Londres 2012 et Jeux olympiques d'hiver de Sochi 2014). Son travail consistait à s'assurer que les athlètes canadiens disposaient toujours d'un accès à des aliments sans danger et efficaces afin qu'ils puissent livrer leurs meilleures performances. Pendant sa conférence, Kelly Anne a décrit les défis propres à la diététique du sport, parlant entre autres des conseils prodigués aux athlètes par des pseudo-nutritionnistes, mais également les incroyables occasions qui s'offrent aux diététistes canadiens intéressés par la nutrition du sport.

Le leadership visionnaire et l'esprit novateur dans le domaine de la nutrition du sport de Kelly Anne, notre fière récipiendaire du prix commémoratif Ryley-Jeffs, constituent sans aucun doute un exemple de la vision de Violet Ryley et de Kathleen Jeffs. Félicitations Kelly Anne!

Consultez le présent numéro de la Revue pour lire les résumés des recherches en diététique de la FCRD présentées au congrès de cette année.

(Rev can prat rech diétét. 2015; 76:3)

(DOI: 10.3148/cjdpr-2015-023)

Publié au dcjournal.ca le 17 août 2015.
2015-2016

Editorial

Board

Comité

de rédaction

$$
\text { Marcia Cooper, PhD, RD }
$$

Ottawa, ON

Wendy Dahl, PhD, RD,

Gainesville, Florida
Karen Davison, $\mathrm{PhD}$, RD

Mission, BC

Heather Keller PhD, RD, Waterloo, $\mathrm{ON}$
Daphne Lordly, DEd, PDt

Halifax, NS

Hugues Plourde, PhD, RD,

Montreal, QC 